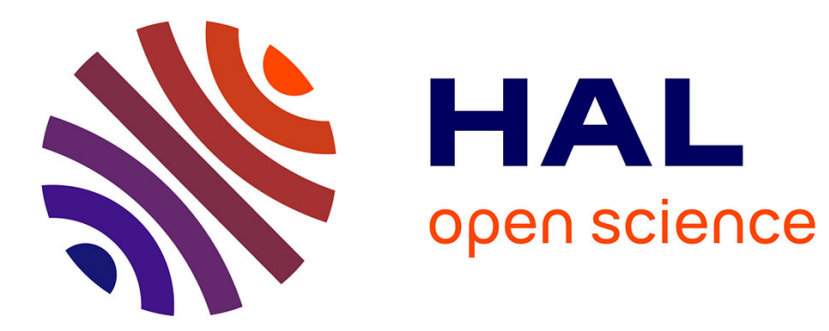

\title{
Évaluation des services écosystémiques urbains : de la rhétorique à la pratique. L'apport de l'approche par habitat
}

\author{
Wissal Selmi, Christiane Weber
}

\section{To cite this version:}

Wissal Selmi, Christiane Weber. Évaluation des services écosystémiques urbains: de la rhétorique à la pratique. L'apport de l'approche par habitat. Environnement Urbain / Urban Environment, 2017. hal-01887854

\section{HAL Id: hal-01887854 \\ https://hal.science/hal-01887854}

Submitted on 4 Oct 2018

HAL is a multi-disciplinary open access archive for the deposit and dissemination of scientific research documents, whether they are published or not. The documents may come from teaching and research institutions in France or abroad, or from public or private research centers.
L'archive ouverte pluridisciplinaire HAL, est destinée au dépôt et à la diffusion de documents scientifiques de niveau recherche, publiés ou non, émanant des établissements d'enseignement et de recherche français ou étrangers, des laboratoires publics ou privés. 


\section{EUE \\ Environnement Urbain / Urban Environment}

Volume 11 | 2017

Les espaces verts urbains : éclairages sur les services écosystémiques culturels

\section{Évaluation des services écosystémiques urbains :} de la rhétorique à la pratique. L'apport de l'approche par habitat

\section{Wissal Selmi and Christiane Weber}

\section{OpenEdition}

\section{Journals}

Electronic version

URL: http://journals.openedition.org/eue/1799

ISSN: 1916-4645

\section{Publisher}

Institut national de Recherche scientifique Urbanisation Culture et Société

Brought to you by Institut national de recherche en sciences et technologies pour l'environnement et l'agriculture (IRSTEA)

irstea

\section{Electronic reference}

Wissal Selmi and Christiane Weber, «Évaluation des services écosystémiques urbains : de la rhétorique à la pratique. L'apport de l'approche par habitat », Environnement Urbain / Urban Environment [Online], Volume 11 | 2017, Online since 24 July 2017, connection on 04 October 2018. URL : http:// journals.openedition.org/eue/1799

\footnotetext{
This text was automatically generated on 4 October 2018.

(c) Institut National de Recherche Scientifique Centre Urbanisation Culture et Société
} 


\title{
Évaluation des services écosystémiques urbains : de la rhétorique à la pratique. L'apport de l'approche par habitat
}

\author{
Wissal Selmi and Christiane Weber
}

\section{Introduction et contexte général}

1 L'évaluation des écosystèmes naturels et semi-naturels prend sa source dans le vif débat philosophique de l'éthique environnementale centrée sur le rapport Humain-Nature et la valeur de cette dernière (Larrère, 1997). En se basant sur une approche écocentrique, cette valeur est dite intrinsèque (ou non instrumentale). Elle renvoie à l'existence des écosystèmes et au fonctionnement écologique qui maintient la survie de ces composantes naturelles. Elle se définit comme une valeur propre et indépendante des besoins et des profits humains (Hawkins, 2003 ; Tientenberg et al., 2013). La valeur de la Nature est a contrario dite instrumentale quand elle est approchée avec une vision anthropocentrique. Elle est considérée comme un support essentiel à la survie de la société humaine. Cette posture a appuyé l'utilisation du concept de services écosystémiques (SE) dans les années 1970. Tributaire d'un aspect sociétal, le concept se définit comme l'ensemble des bénéfices directs et indirects issus du fonctionnement des écosystèmes (DeGroot et al., 2002). Bien que les principes qui régissent ces deux visions ne soient pas les mêmes, elles se rejoignent dans la mesure où elles sont toutes les deux réductrices quant à la valeur des écosystèmes naturels qui sont, dans les courants de pensée extrêmes, soient des écosystèmes suprêmes (c'est-à-dire le courant de pensée deep ecology) (Sauveur, 2010) soient des biens marchands (Norton, 1997). Face à ce constat, le développement d'une vision combinant les deux précédentes est devenu essentiel pour appréhender les différentes facettes des éléments naturels. Une telle approche duale peut permettre non seulement de quantifier et d'optimiser les SE mais aussi d'étudier et de 
comprendre le fonctionnement écologique sous-jacent des écosystèmes naturels et seminaturels pour en assurer la pérennité.

Ce cadre conceptuel d'évaluation est empreint d'incertitudes et d'approximations. La confusion entre le concept de "fonction» et celui de "service» (souvent considéré comme synonymes) en est responsable. En effet, le terme fonction ou «multifonction » est utilisé dans la plupart des cas avec un sens sociétal qui renvoie au bien-être humain : fonction de détente, fonction de récréation, fonction esthétique qui correspondent à des bénéfices rendus à la société humaine.

3 Pour clarifier, il est nécessaire de rappeler le lien de causalité complexe entre le terme fonction et le terme service, en soumettant le premier à une interprétation purement écologique (en étudiant la composition, la structure, les processus génétiques, etc.) et en attribuant au second une dimension sociétale (Bastian et al., 2012). Cette démarche convoque à la fois les dimensions écologique et utilitariste. La précision de ce lien causal entre fonction et service a été explicitée dans les années 90 (de Groot et al., 1992, 2002), il a été ensuite illustré par la notion « service cascade » dans les années 2010 (de Groot et al., 2010 ; Potschin et Haines-Young, 2011). La particularité de cette schématisation réside dans son explicitation des différents éléments allant d'éléments producteurs des SE jusqu'aux bénéficiaires. Bien que le "service cascade " convoque, en apparence, une approche simpliste, il n'exclut pas la complexité des liens, car il établit les flux de rétroaction et prend en compte l'irréversibilité des effets.

Depuis presque 40 ans, les études portées sur l'évaluation des écosystèmes naturels et plus particulièrement des services écosystémiques ont eu recours à une approche économique, car cette dernière fournit des valeurs monétaires relativement faciles à intégrer dans le processus de prise de décision. Ainsi, les SE deviennent un avatar économique et connaissent alors un réel succès dans les travaux en économie de l'environnement. Néanmoins, ce concept gagne en pertinence dès lors qu'il est appréhendé de manière transversale: soit sociale, si l'on cherche à déterminer les attentes des usagers; soit économique, lorsqu'on souhaite quantifier un coût ou un bénéfice dans le cas d'une évaluation environnementale par exemple lors d'implantation d'infrastructure ; soit écologique, si l'on cherche à quantifier le potentiel des écosystèmes à contribuer au bien-être humain. Or ce dernier aspect est souvent ignoré (De Groot et al., 2010). Pourtant de nombreuses études ont démontré que l'évaluation écologique permet d'étudier à la fois le fonctionnement des écosystèmes naturels et semi-naturels et de quantifier les services rendus à la société (Gomez-Baggethun et al., 2013).

5 À partir des années 1990, les scientifiques ont commencé à interroger la place du milieu urbain et de ses espaces à caractère naturel (connus sous le nom d'espaces végétalisés urbains) dans le champ de la protection et de la valorisation de la biodiversité et de l'optimisation des SE. Ce faisant, l'appréhension des interactions entre les êtres humains et leur environnement s'est trouvée requestionnée. La ville devient, dès lors, un terrain d'expérimentation de prédilection pour les chercheurs et les dualités « ville/nature » et « humain/nature » et se trouve en tête des questionnements scientifiques : la biodiversité en ville conserve-t-elle les mêmes fonctions et services que la biodiversité patrimoniale ? Devons-nous utiliser les mêmes approches d'évaluation pour appréhender sa valeur? Les espaces à caractère naturel en ville se réduisent-ils à de simples éléments esthétiques ou apportent-ils d'autres bénéfices? 
6 L'ensemble de ces constats a conduit à l'élaboration du cadre conceptuel suivant (Bastian et al., 2012; Haines-Young et Potschin, 2010 et de Groot et al., 2010): les espaces végétalisés urbains (EVU) sont définis comme l'ensemble des formations à caractère naturel regroupant des formations végétales très variées. Ces espaces sont multifonctionnels au sens écologique du terme, c'est-à-dire qu'ils effectuent un ensemble de fonctions écologiques qui peuvent être étudiées à travers leur composition, leur structure ou alors leurs processus écologiques (comme la productivité, les flux d'énergie, le dépôt sec, etc.). L'ensemble de ces fonctions produit des services écosystémiques (SE) définis comme des bénéfices directs. Le terme services est souvent associé à un autre terme celui des "desservices" qui correspond aux effets néfastes produits par les écosystèmes naturels et semi-naturels. Ces bénéfices et ces desservices agissent sur le bien-être humain (services de régulation, service de récréation, services esthétiques, etc.) et peuvent être évalués à travers des approches d'évaluation écologiques, sociologiques, économiques, etc. Les liens entre ces différentes composantes (EVU, fonction, services, valeur) sont complexes. Par exemple, l'évaluation d'un service écosystémique donné peut intervenir sur le processus décisionnel soit pour l'optimiser et/ou pour favoriser les démarches de protection de la formation végétale étudiée.

7 Dans cet article, nous nous focalisons sur l'évaluation de la valeur instrumentale des espaces végétalisés urbains, en termes biophysique. Comment réussir l'évaluation d'une telle valeur en étudiant à la fois les fonctions et les services écosystémiques ? Ce questionnement vise une approche opérationnelle qui met en pratique un concept controversé.

8 L'objectif de cet article, après avoir exposé dans la deuxième partie les différentes approches d'évaluation des services écosystémiques urbains, est d'illustrer ensuite l'évaluation des services rendus par les arbres urbains à Strasbourg. La quatrième partie est consacrée aux perspectives scientifiques et opérationnelles associées à cette approche d'évaluation.

\section{Approches d'évaluation}

9 L'évaluation des services écosystémiques issus du fonctionnement des écosystèmes seminaturels urbains reste une approche complexe (James et al., 2009 ; Bastian et al., 2012). La première apparition des modèles d'évaluation économique des SE date des années 1970 (de Groot, 2003, Teyssèdre, 2010). Les chercheurs se sont concentrés sur une estimation monétaire des bénéfices en faisant appel à diverses méthodes comme le prix hédoniste, le coût de transport, le prix du marché, etc. En revanche, si l'on considère les potentiels biophysiques, écologiques et socioéconomiques illustrés par Pickett et al (2008), c'est plutôt une approche transversale plus englobante qui serait recommandée (James et al., 2009 ; Jim, 2011). James et al. (2009) avancent l'intérêt et la difficulté d'introduire des logiques et des approches pluridisciplinaires aussi bien pour examiner le fonctionnement de tels écosystèmes que pour quantifier et évaluer les SE (James et al., 2009). Notre synthèse de références bibliographiques isole deux approches pour étudier les services écosystémiques rendus à la société. 


\subsection{Approche ascendante par habitat}

10 En dépit de son importance, cette approche est très peu développée (de Groot et al., 2010). Elle consiste à (1) caractériser les habitats, (2) évaluer leur potentiel écologique à travers l'étude de leur fonctionnement et (3) orienter les pratiques d'aménagement et de gestion vers une optimisation de production des services écosystémiques (Haines-Young et Potschin, 2006). L'avantage de cette approche est qu'elle traite aussi de l'impact sur la production des SE des facteurs anthropiques telles la gestion et l'artificialisation des terres (de Groot et al., 2010 ; Dobbs et al., 2011). Cette approche, appliquée aux milieux naturels, se focalise souvent sur les écosystèmes sanctuaires à forte valeur écologique (Defra, 2007). Elle conduit à interroger la mise en application d'une telle évaluation pour des écosystèmes semi-naturels « ordinaires » affectés par des pressions anthropiques.

\subsection{Approche descendante par services écosystémiques}

11 L'approche descendante, couramment utilisée, se focalise sur l'identification des formations végétales capables de fournir certains services écosystémiques (Haines-Young et Potschin, 2006). Les SE sont sélectionnés d'une manière non exhaustive à partir de travaux scientifiques. Le choix des SE, lorsqu'il se réfère aux intérêts sociopolitiques, se fait par exemple en fonction des objectifs de planification. Les chercheurs, dans ce cas, privilégient le recours à des enquêtes auprès des services compétents (planificateurs ou gestionnaires) pour (1) caractériser leurs motivations et leurs pratiques (ex. Young 2010), (2) identifier les SE les plus pertinents pour une communauté d'acteurs (ex. Defra, 2007). Cette démarche, si elle favorise l'échange entre la sphère des scientifiques et celle des décideurs, n'examine cependant pas le fonctionnement des écosystèmes naturels et seminaturels.

Qu'il s'agisse d'une approche ascendante ou descendante, les scientifiques ont développé un large éventail d'indicateurs portant sur : (1) la valeur monétaire (ex. coût de transport pour quantifier le service de récréation des espaces verts) (2) l'impact des SE sur le bienêtre social (ex. l'évaluation contingente pour évaluer les aménités) et (3) l'effet physicochimique sur l'environnement (ex. le flux des polluants $\left(\mathrm{g} / \mathrm{cm}^{2} / \mathrm{s}\right)$ pour quantifier la purification de l'air) (Gomez-Baggethun et al., 2013 ; Dobbs et al., 2011)

13 Étant donné que l'évaluation des services écosystémiques rendus par les espaces végétalisés urbains est une thématique récente, l'approche ascendante par habitat a été choisie pour fournir des réponses préliminaires sur le potentiel de la végétation à produire des services écosystémiques.

\section{2. Étude de cas : mise en place d'une approche par habitat à l'échelle de la ville de Strasbourg}

14 L'objectif de cet article est de présenter une méthode d'évaluation écologique des services écosystémiques rendus par la végétation urbaine. Cet objectif principal se décline en un objectif spécifique : démontrer que l'approche écologique peut éventuellement apporter un regard nouveau sur les modes d'évaluation, c'est-à-dire la quantification de ces services à travers les processus écologiques de la végétation urbaine. Pour ce faire, une quantification des services écosystémiques rendus par les arbres urbains à Strasbourg a 
été effectuée à l'aide du modèle i-Tree Eco (Nowak et al., 2008). Développé dans les années 1990 par l'«US departement of agriculture », i-Tree Eco évalue la structure la végétation arborée et quantifie les services écosystémiques rendus à la société. Considéré comme l'outil le plus complet pour traiter ce sujet (Chapparo and Terradas, 2009), le modèle a été utilisé dans des villes européennes comme Barcelone en Espagne (Chapparo and Terradas, 2009 ; Baro et al., 2014) ; Torbay en Grande-Bretagne (Rogers et al, 2011), Florence en Italie (Paoletti et al., 2011), etc.

15 À noter que l'évaluation monétaire des SE proposée par le modèle n'est pas abordée dans le cadre de cet article.

\subsection{Terrain d'étude}

16 Le modèle a été appliqué à l'échelle de la ville de Strasbourg (Chef-lieu de la région Alsace-Champagne-Ardenne-Lorraine). Elle s'étend sur une superficie de $78.3 \mathrm{~km}^{2}$ et compte environ 274394 habitants (recensement INSEE de 2012). La ville dispose en sa périphérie de trois massifs forestiers alluviaux : la forêt de la Robertsau au nord (493 ha), la forêt du Neuhof ( 757 ha) et l'île de Rohrschollen (309 ha) au sud de la ville (Figure 20) (CUS, 2013). Dans le cadre de cette étude, seuls les espaces gérés par les services publics sont étudiés, car ces derniers pourraient faire l'objet d'une intervention de la part des décideurs pour la mise en place de la future trame verte urbaine. Nous nous intéressons plus particulièrement aux formations arborées qui peuvent aller des plantations d'arbres isolées à des formations très développées (haies, boisement, alignements, etc.).

\section{2 Évaluation des services écosystémiques rendus par les arbres}

17 La particularité d'i-Tree Eco réside dans son approche ascendante : il fait le lien entre la structure des arbres (espèces; classes de DHP : diamètre à hauteur de poitrine; état sanitaire, biomasse, surface foliaire, etc.), son fonctionnement et les services qu'elle procure à la société (stockage et séquestration du carbone; élimination des polluants atmosphériques, production de l'oxygène). Il permet ainsi de comprendre les interactions entre l'air ambiant, les microclimats et la végétation arborée en prenant en compte ses processus physiologiques (ex. en étudiant le processus de dépôt sec pour quantifier le service d'élimination des polluants). Pour ce faire, il se base sur l'association des données numériques et spatiales (climatologiques, atmosphériques, d'occupation du sol, etc.) et des mesures dendrométriques de terrain (nombre d'arbres, nombre d'espèces, diamètre, hauteur, dépérissement, état sanitaire, etc.).

\subsubsection{Collecte des données numériques}

L'ensemble des données numériques a été collecté auprès des organismes spécialisés (institut national de l'information géographique et forestière-IGN, services municipaux, agence pour la surveillance de la qualité de l'air, Météo France, etc.). Suivant les recommandations prescrites par le manuel du modèle, les données climatologiques et atmosphériques ont été collectées sur une période de 12 mois (Tableau n²). Ces données sont par la suite entrées dans le modèle i-Tree. 
Tableau 1. Données requises pour le modèle i-Tree Eco (Selmi, 2014)

\begin{tabular}{|c|c|c|}
\hline Données & Sources & Objectifs \\
\hline $\begin{array}{l}\text { Concentrations } \\
\text { horaires des } \\
\text { polluants } \\
(2012 / 2013)\end{array}$ & $\begin{array}{l}\text { Association pour la } \\
\text { surveillance et l'étude de } \\
\text { la pollution } \\
\text { atmosphérique en Alsace } \\
\text { (ASPA) }\end{array}$ & $\begin{array}{l}\text { Calcul du dépôt sec des polluants suivants: } \\
\text { ozone }\left(\mathrm{O}_{3}\right) \text {; dioxyde de soufre }\left(\mathrm{SO}_{2}\right) \text {; dioxyde } \\
\text { d'azote }\left(\mathrm{NO}_{2}\right) \text {; monoxyde de carbone }(\mathrm{CO}) \text {; } \\
\text { particules en suspension dont le diamètre est } \\
\text { entre } 2.5 \text { et } 10 \text { micromètres }\left(\mathrm{PM}_{10} \text { coarse) et }\right. \\
\text { particules en suspension dont le diamètre est } \\
\text { inférieur à } 2.5\left(\mathrm{PM}_{2.5}\right) \text {. }\end{array}$ \\
\hline $\begin{array}{l}\text { Données } \\
\text { climatologiques } \\
(2012 / 2013)\end{array}$ & $\begin{array}{l}\text { Météo France } \\
\text { National Oceanic and } \\
\text { Atmospheric Administration } \\
\text { (NOAA) }\end{array}$ & $\begin{array}{l}\text { Ajustement des algorithmes en fonction des } \\
\text { conditions locales. }\end{array}$ \\
\hline $\begin{array}{l}\text { Données } \\
\text { phénologiques }\end{array}$ & $\begin{array}{l}\text { L'Observatoire } \quad \text { Des } \\
\text { Saisons, GDR CNRS } 2968\end{array}$ & $\begin{array}{l}\text { Détermination des événements périodiques de } \\
\text { feuillaison et de la chute des feuilles qui } \\
\text { définissent le cycle biologique des arbres. }\end{array}$ \\
\hline Données spatiales & $\begin{array}{l}\text { BD TOPO } 2008 \\
\text { BD CIGAL } 2008 \\
\text { BD espaces verts } 2010 \\
\text { BD SERTIT } 2012\end{array}$ & $\begin{array}{l}\text { Délimitation du terrain d'étude et génération } \\
\text { de l'échantillon; } \\
\text { Identification de l'occupation du sol; } \\
\text { Détermination du couvert arboré. }\end{array}$ \\
\hline $\begin{array}{l}\text { Données } \\
\text { dendrométriques }\end{array}$ & $\begin{array}{l}\text { Campagne } \\
\text { d'échantillonnage }\end{array}$ & $\begin{array}{l}\text { Prise en compte des caractéristiques de la } \\
\text { végétation arborée locale. }\end{array}$ \\
\hline
\end{tabular}

\subsection{2 Échantillonnage et collecte des données dendrométriques}

Les données dendrométriques ont été collectées en suivant un protocole d'échantillonnage spécifique. Un échantillon représentatif de la végétation arborée et stratifié en fonction de huit classes d'occupation du sol a été généré (Figure $\mathrm{n}^{\circ} 1$ ). La réalisation du plan d'échantillonnage s'est basée sur les quatre étapes suivantes: délimitation des espaces végétalisés publics gérés par les services municipaux; stratification de ces espaces en fonction des classes d'occupation du sol, détermination du couvert arboré dans les espaces végétalisés publics; génération de l'échantillon et collecte de données. 


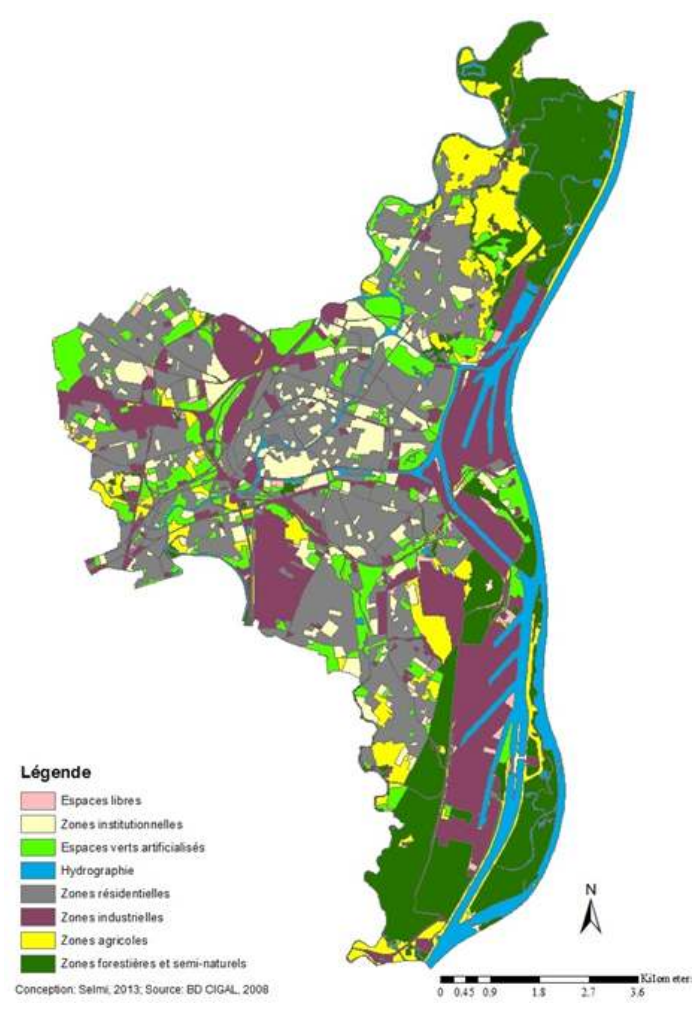

FigURE 1. STRATIficATION DE LA VILLE DE STRASBOURg EN FONCTION DE CLASSES D'OCCUPATION DU SOL

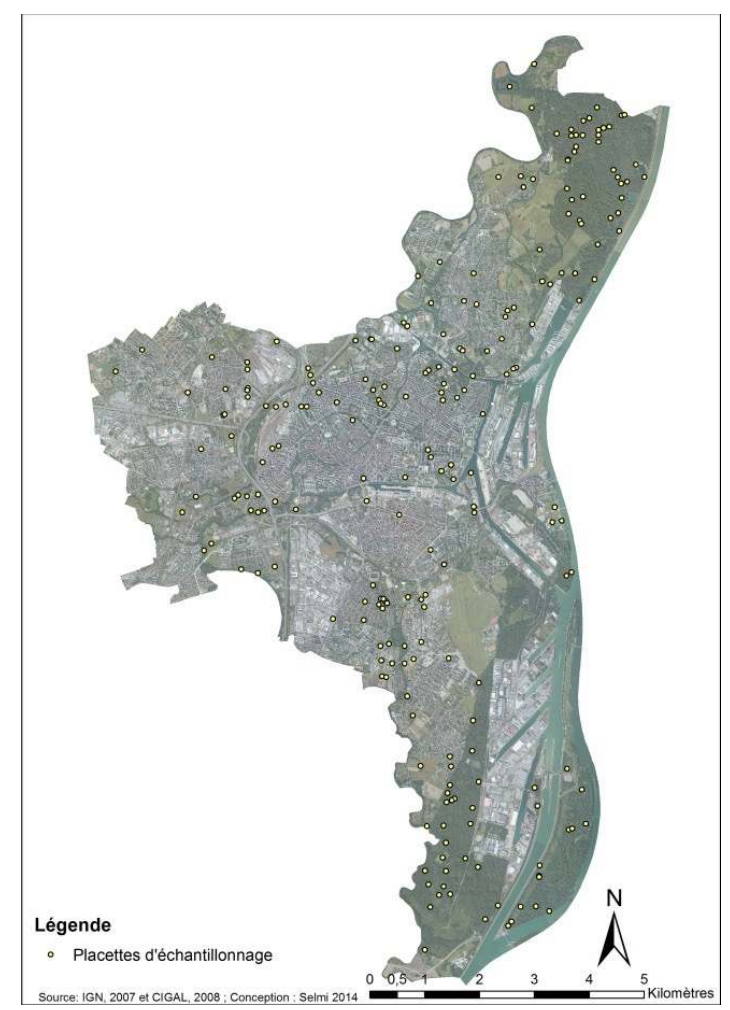

FIgURE 2. DISTRIBUTION DES PLACETTES d'ÉCHANTILLONNAgE

20 À l'issue de la superposition des jeux de données spatiales, 233 placettes de $400 \mathrm{~m}^{2}$ de surface ont été distribuées aléatoirement au niveau des espaces végétalisés publics de la ville de Strasbourg (Figure $\mathrm{n}^{\circ} 2$ ). Une fois localisées à travers leurs coordonnées 
géographiques, les placettes ont fait l'objet d'une campagne d'échantillonnage qui s'est déroulée pendant la saison estivale de l'année 2013.

21 Les données collectées renseignent sur l'occupation du sol, le couvert végétal, la distance entre les pieds d'arbres et le centre de la placette. Pour chaque pied d'arbre localisé dans les placettes, l'espèce a été identifiée puis une série de mesures dendrométriques a été effectuée : le diamètre, la hauteur, la projection de la couronne, le facteur d'exposition à la lumière, le pourcentage de dépérissement, etc. Les données collectées ont servi pour calculer la surface et la biomasse foliaire et d'ajuster la modélisation en fonction des caractéristiques de la végétation locale.

À l'issue de ce travail, 2033 arbres ont été inventoriés dans ces espaces végétalisés publics.

\subsection{Résultats}

Pour l'évaluation écologique du potentiel des arbres à produire des SE, le modèle i-Tree Eco fournit deux types d'indicateurs :

\subsubsection{Indicateurs d'état de la végétation}

24

Les indicateurs d'état mettent l'accent sur l'état de la végétation arborée et son aspect structurel. Le modèle estime que la ville de Strasbourg compte environ 588000 arbres (en incluant les trois forêts rhénanes) avec une densité de 271 arbres/ha d'espaces végétalisés gérés par les services municipaux de la ville. Les espaces forestiers et semi-naturels renferment $80 \%$ du nombre total des arbres, suivis par la classe d'espaces verts artificiels (parcs, squares, jardins, etc.) (10\%) et les espaces institutionnels (centres scolaires et culturels, centres hospitaliers) (3\%).

L'espèce la plus commune à l'échelle de la ville est le hêtre commun (12\%) suivi par le noisetier commun ( $11 \%$ ) et le frêne élevé (Figure $n^{\circ} 3$ ). En revanche, en excluant les trois forêts rhénanes, l'érable sycomore arrive au premier rang suivi par l'érable plane et le frêne élevé. 


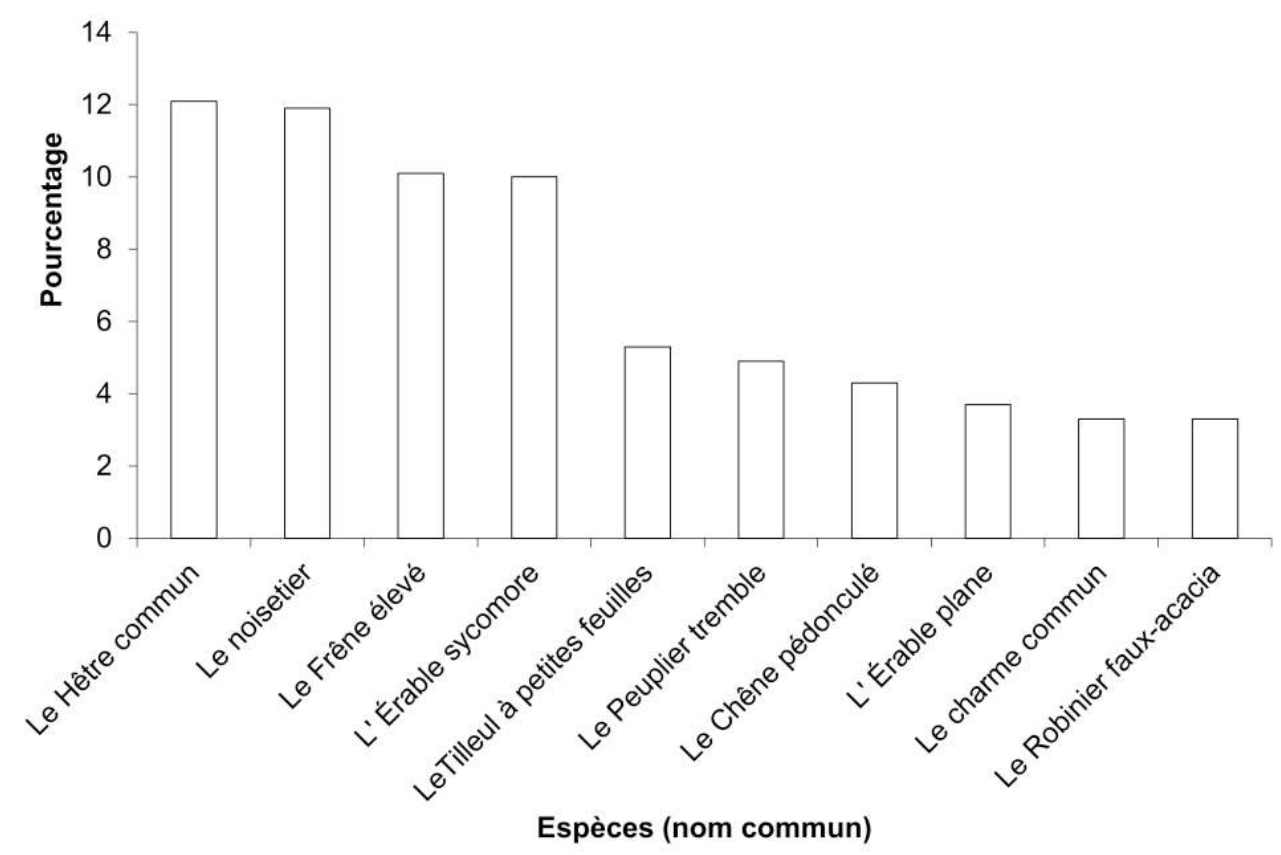

Figure 3. Les espèces communes dans les espaces végétalisés urbains de Strasbourg (Selmi, 2014)

Le patrimoine arboré strasbourgeois est composé d'arbres de différentes tailles avec un diamètre à la hauteur de la poitrine (DHP) très varié. Il varie entre une valeur minimale de $1,6 \mathrm{~cm}$ et dans certains cas plus de $100 \mathrm{~cm}$. Les arbres dont le DHP est inférieur à 15.2 $\mathrm{cm}$ regroupent environ $44 \%$ de la population d'arbres. La majorité des arbres larges (dont le diamètre est supérieur ou égal à $54 \mathrm{~cm}$ ) sont concentrés dans des zones agricoles $(30 \%)$ et libres (22\%) tandis que $49 \%$ des arbres localisés dans les forêts ont un DHP inférieur ou égal à $15.2 \mathrm{~cm}$. Le graphique ${ }^{\circ} 4$ montre la distribution des fréquences des classes de DHP, celle-ci varie d'une faç on significative selon le type d'occupation du sol. Ces variations s'expliquent par la densité d'arbres qui varie d'une classe d'occupation du sol à une autre : plus elle est importante, plus l'accès à la lumière est réduit et plus la taille est petite (c'est le cas des zones forestières) (McPherson, 1998). 


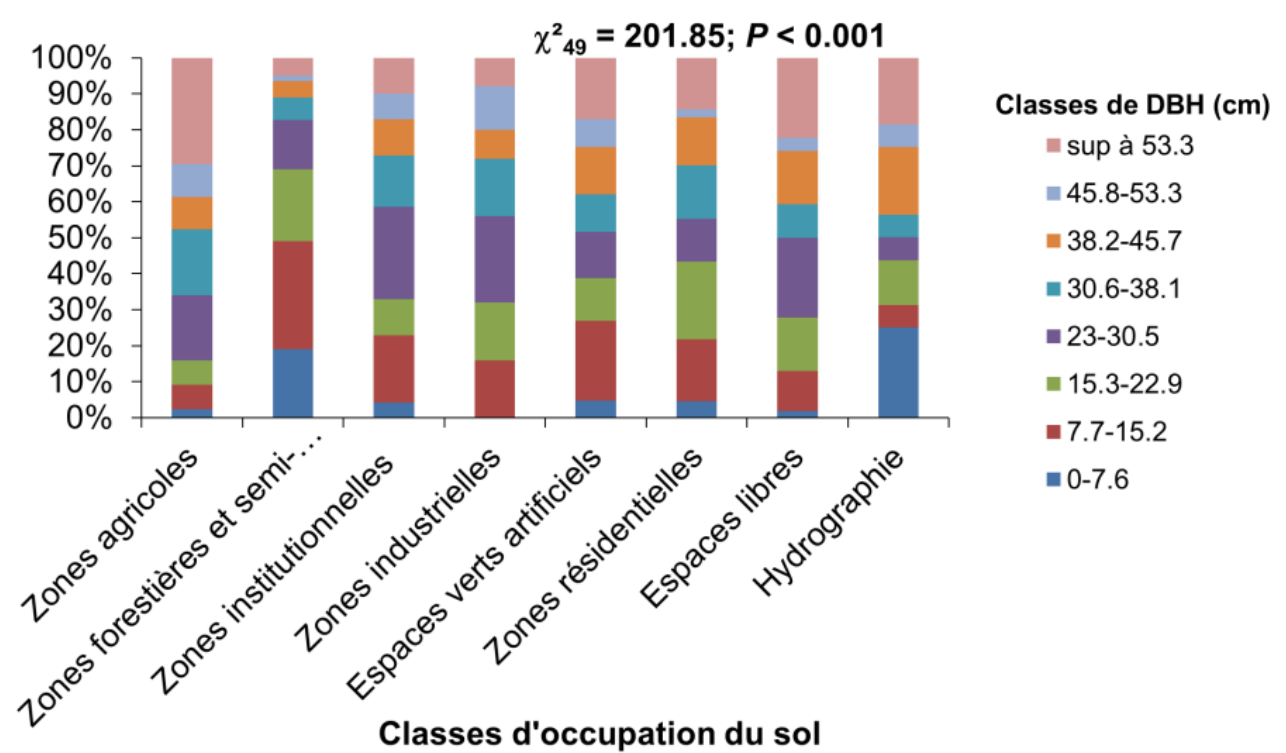

Figure 4. Variation de la composition de la végétation en termes de classes de DHP selon le type d'occupation du sol (Selmi, 2014)

27 Le modèle estime que la surface foliaire au sein des espaces végétalisés municipaux représente environ $91 \mathrm{~km}^{2}$ et la biomasse foliaire est de l'ordre de 6560 tonnes. Les analyses de variance et le test de Duncan ont montré que ces estimations varient significativement en fonction des classes d'occupation du sol (Figure n5). Ces variations sont expliquées par le nombre et la densité des arbres ainsi que le taux de couvert arboré.
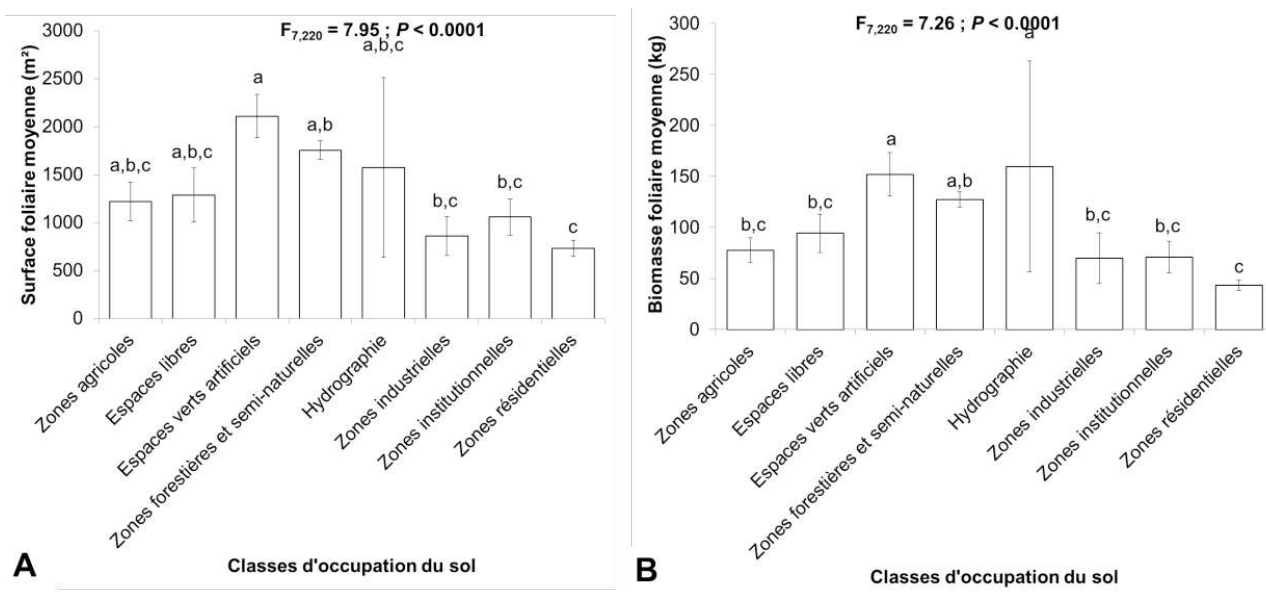

Figure 5. Les résultats de l'ANOVA vérifiant l'effet des classes d'occupation du sol sur la surface foliaire (A) et la biomasse foliaire (B), les barres d'erreur qui correspondent à l'erreur standard de la moyenne. a, b et c sont les groupes générés à partir de test de Duncan (Selmi, 2014).

Outre les indicateurs d'état, le modèle a estimé qu'environ $15 \%$ de la surface de la ville de Strasbourg est susceptible d'accueillir des nouvelles plantations. Cet espace (à planter) s'étale sur $38 \%$ de la surface des zones libres, $12 \%$ de la surface des zones résidentielles et $41 \%$ au niveau des zones agricoles (les espaces non cultivés).

\subsubsection{Indicateurs de performance}

Les indicateurs de performance estimés par le modèle i-Tree et basés sur les données de terrains et les données locales ont montré que les arbres gérés par les services 
municipaux stockent environ 128000 tC et ont séquestré entre juillet 2012 et juin 2013 environ $4059 \mathrm{tC} / \mathrm{an}$. Outre l'occupation du sol, la capacité des arbres à stocker et à séquestrer le carbone varie en fonction d'autres facteurs comme la biomasse, la surface foliaire et le diamètre.

À titre d'exemple, la moyenne du stockage du carbone est de l'ordre de $5700 \mathrm{~kg}$ pour un arbre ayant un DHP compris entre 106 et $114 \mathrm{~cm}$ et de $19 \mathrm{~kg}$ pour un arbre ayant un DHP compris entre 7.6 et $15.2 \mathrm{~cm}$. La séquestration annuelle brute pour les mêmes dimensions est de l'ordre de $51 \mathrm{~kg} / \mathrm{an}$ et $2 \mathrm{~kg} / \mathrm{an}$. La figure $\mathrm{n}^{\circ} 6$ montre la différence de stockage total et la séquestration annuelle (brute et nette) du carbone par arbre en fonction du DHP. Les deux services augmentent avec l'accroissement de la taille des arbres c'est-à-dire pendant la phase de la croissance. Étant plus sensible à l'âge et à la taille des arbres (Nowak, 1994), le taux de séquestration annuelle du carbone commence à baisser à partir d'un DHP égal à $99 \mathrm{~cm}$ alors que le taux de stockage diminue à un DHP de $129 \mathrm{~cm}$.

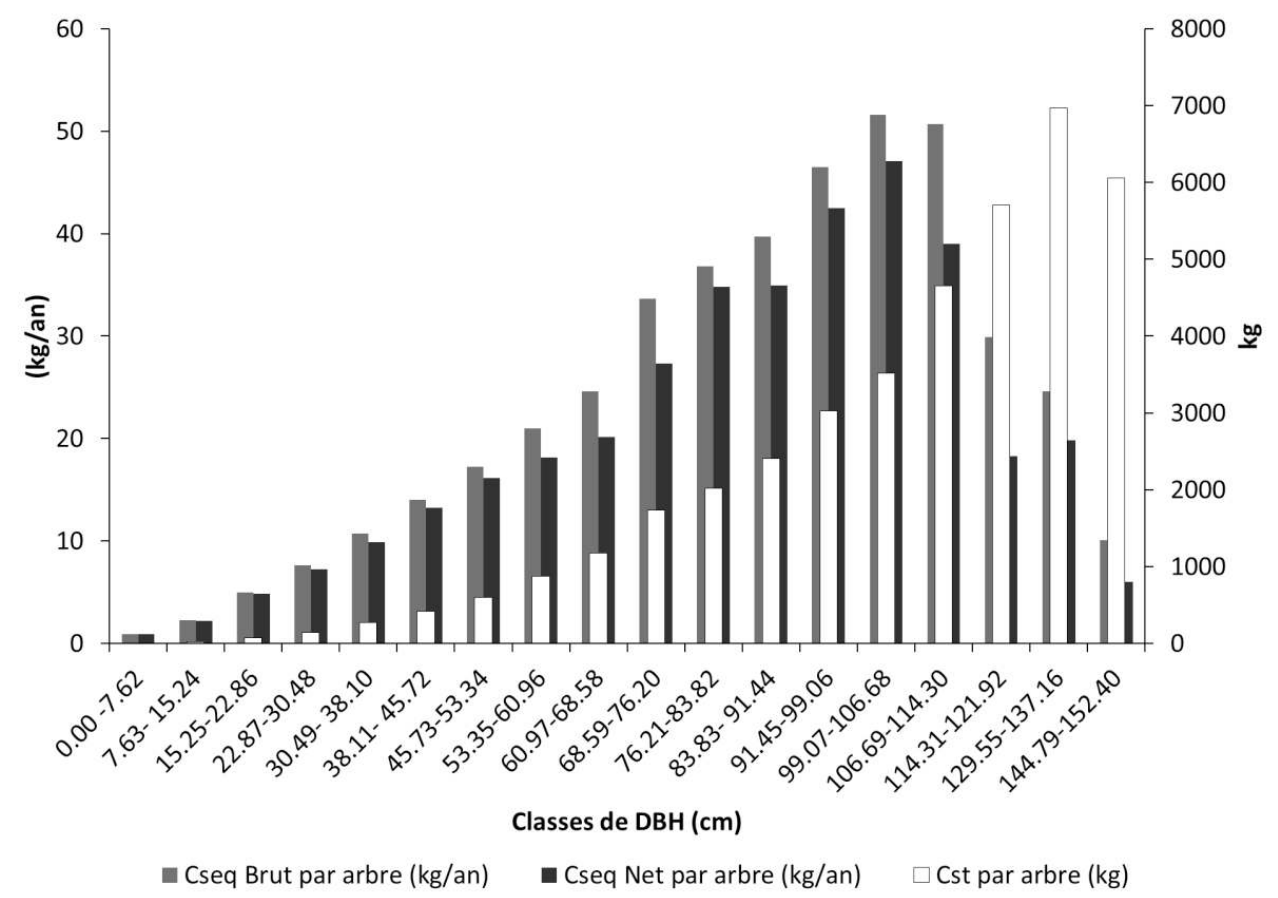

Figure 6. Variation de stockage et de séquestration du carbone par pied d'arbre et par classe de DHP à Strasbourg (Selmi, 2014)

La variation de la séquestration du carbone en fonction du DHP peut aussi s'illustrer par un modèle de régression linéaire issue d'une transformation logarithmique d'un modèle puissance appliqué à l'échelle de notre échantillon (Figure $n^{\circ} 7$ ). Ce dernier montre que les séquestrations, nette $(\beta=1.23 \pm 0.04, t=31.33 ; P<0.0001)$ et brute $(\beta=2.19 \pm 0.05, t=42.60$; $\mathrm{P}<0.0001)$ sont corrélées positivement au DHP avant qu'il n'atteigne $107 \mathrm{~cm}$. Au-delà de ce diamètre, la tendance s'inverse. Dans ce cas, le taux de séquestration du carbone diminue considérablement et devient corrélé négativement avec le DHP (net: $\beta=-5.37$ $\pm 1.77, \mathrm{t}=-3.04 ; \mathrm{P}=0.0934 ;$ brut $: \beta=-4.93 \pm 0.99, \mathrm{t}=-5.04 ; \mathrm{P}=0.0372$ ). 

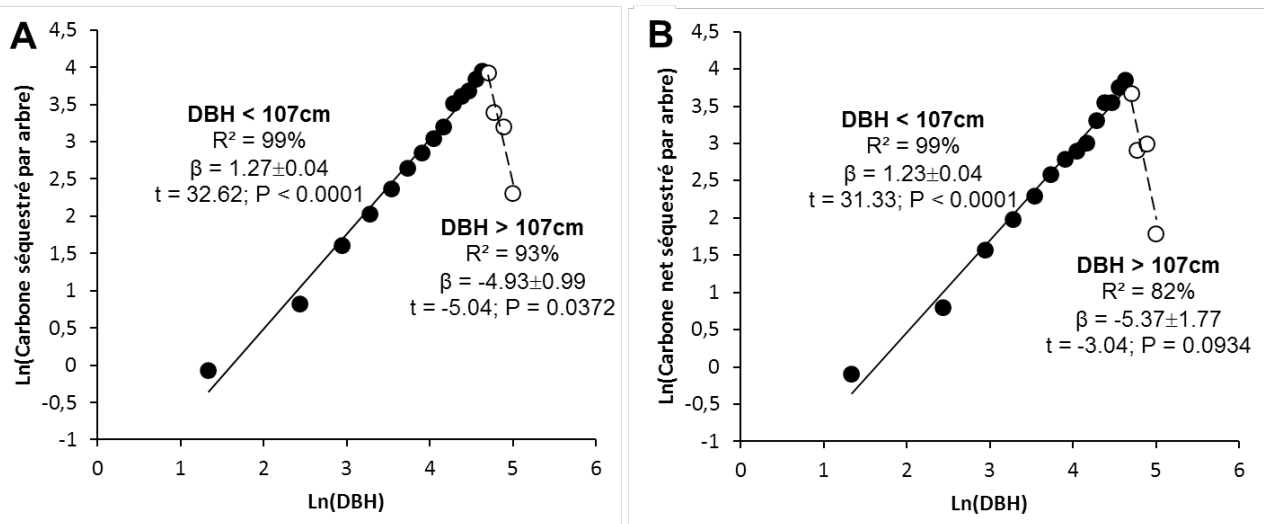

Figure 7. Modèle linéaire suite à une transformation logarithmique montre le changement de séquestration du carbone brut (A) et net (B) en fonction des classes de DHP (variable explicative) (Selmi, 2014).

Une analyse de la covariance (ANCOVA) a permis de confirmer la différence de taux de séquestration du carbone à valeur de DHP égale entre deux classes d'occupation du sol (zones résidentielles et zones forestières) (Tableau $n^{\circ} 3$ et Figure $n^{\circ} 8$ ).

Tableau 3. Résultats de l'ANCOVA du taux annuel de séquestration du carbone (variable dépendante) en fonction du DHP (variable explicative continue) et du type d'occupation du sol (variable explicative catégorielle avec deux classes : zones forestières vs zones résidentielles). $\mathrm{N}=$ 22 (Selmi, 2014).

\begin{tabular}{|l|l|l|l|}
\hline Effet & DDL & F & $P$ \\
\hline Type d'occupation du sol & 1 & 0.39 & 0.5377 \\
\hline DBH & 1 & 270.95 & $<0.0001$ \\
\hline DBH*Type d'occupation du sol & 1 & 7.36 & 0.0143 \\
\hline
\end{tabular}

Comme le montre la figure $n^{\circ} 8$, globalement, le taux de séquestration du carbone augmente avec le DHP. Toutefois, cette augmentation ne se fait pas de la même faç on selon le type d'occupation du sol. En effet la pente de la droite de régression du taux de séquestration du carbone en fonction du DHPest plus important dans le cas des zones résidentielles $\left(\beta \pm S E=0.66 \pm 0.06 ; t_{10}=10.49 ; P<0.0001\right)$ que dans le cas des zones forestières $\left(\beta \pm S E=0.47 \pm 0.03 ; t_{10}=16.95 ; P<0.0001\right)$. En étant dans un espace ouvert, un arbre urbain crôit plus vite qu'un arbre en milieu forestier (couvert fermé avec une forte compétition entre les arbres) et développe une surface foliaire plus importante en milieu urbain qu'en milieu forestier. 


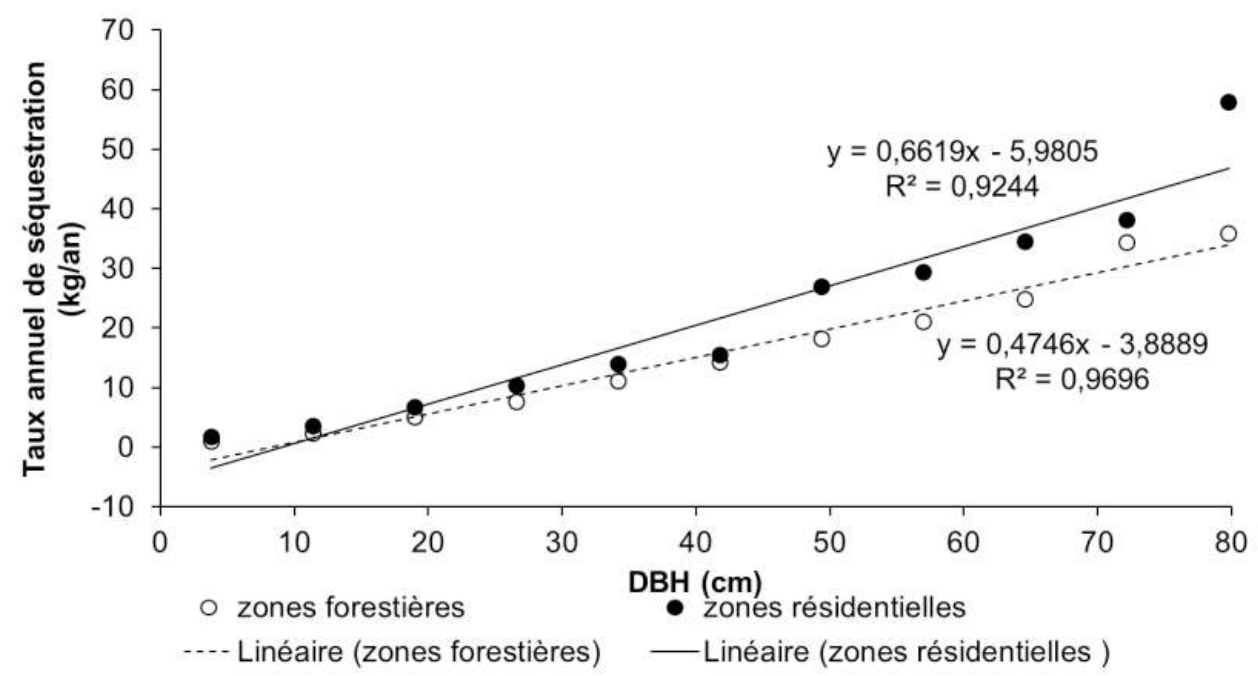

Figure 8. Variation du taux annuel de séquestration du carbone en fonction du DHP dans le cas des zones résidentielles (symboles noirs) et des zones forestières (symboles blancs). Les droites de régression et les équations correspondantes sont également représentées (zones résidentielles : droite continue ; zones résidentielles : droite discontinue) (Selmi, 2014).

Ainsi, outre le couvert arboré, le stockage et la séquestration du carbone sont influencés par la taille et l'état sanitaire du patrimoine arboré. Un pourcentage élevé du couvert arboré et/ou des arbres en phase de croissance et/ou en bon état sanitaire conduisent à un stockage et une séquestration du carbone assez considérable (Nowak et Crane, 2000). Dans le même contexte, un taux de mortalité qui dépasse $5 \%$ convertit le rôle de l'arbre d'un puits de carbone en une source d'émission de carbone (Nowak, 1994).

Durant la période considérée, les arbres ont réussi à éliminer environ $88 \mathrm{t}$ de polluants $\left(1.2 \mathrm{t} /\right.$ an de $\mathrm{CO} ; 13.84 \mathrm{t} / \mathrm{an}$ de $\mathrm{NO}_{2} ; 55.87 \mathrm{t} /$ an de $\mathrm{O}_{3} ; 11.76 \mathrm{t} / \mathrm{an}$ de $\mathrm{PM}_{10}$ coarse $; 4.5 \mathrm{t} / \mathrm{an}$ de $\mathrm{PM}_{2.5}$ et $1.03 \mathrm{t} /$ an de $\mathrm{SO}_{2}$ (Figure $\mathrm{n}^{\circ} 9$ ).

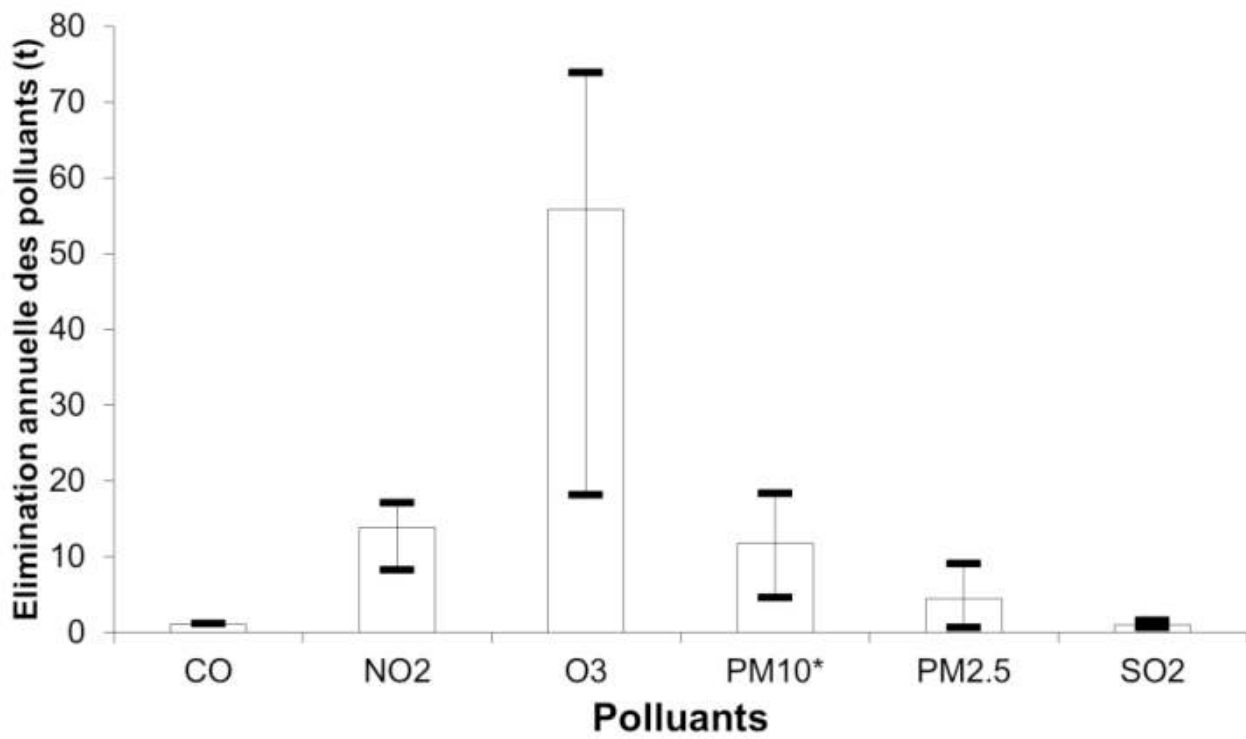

Figure 9. Élimination annuelle des polluants à Strasbourg (les barres correspondent aux éliminations maximale et minimale ; PM10* $=\mathrm{PM}_{10}$ coarse) $($ Selmi, 2014)

Le taux d'élimination des polluants varie en fonction des mois, les pics d'élimination ont été atteints en septembre pour le $\mathrm{CO}(0.26 \mathrm{t} / \mathrm{an})$ et le $\mathrm{NO}_{2}(1.7 \mathrm{t} / \mathrm{an})$, en juin pour l'O $\mathrm{O}_{3}(11$ 
$\mathrm{t} / \mathrm{an})$, le $\mathrm{SO}_{2}(0.17 \mathrm{t} / \mathrm{an})$ et les $\mathrm{PM}_{10}$ coarse $(2.4 \mathrm{t} / \mathrm{an})$ et en juillet pour les $\mathrm{PM}_{2.5}$ (1.02 $\left.\mathrm{t} / \mathrm{an}\right)$ (Figure $\left.\mathrm{n}^{\circ} 10\right)$. Ces variations s'expliquent par la phénologie des arbres, car la surface d'échange gazeux entre l'atmosphère et les arbres est plus importante pendant la saison estivale ce qui correspond à une hausse de dépôt sec des polluants. Par ailleurs, l'augmentation des concentrations des polluants dans l'atmosphère pendant l'été peut aussi expliquer l'accroissement d'élimination des polluants pendant cette période.

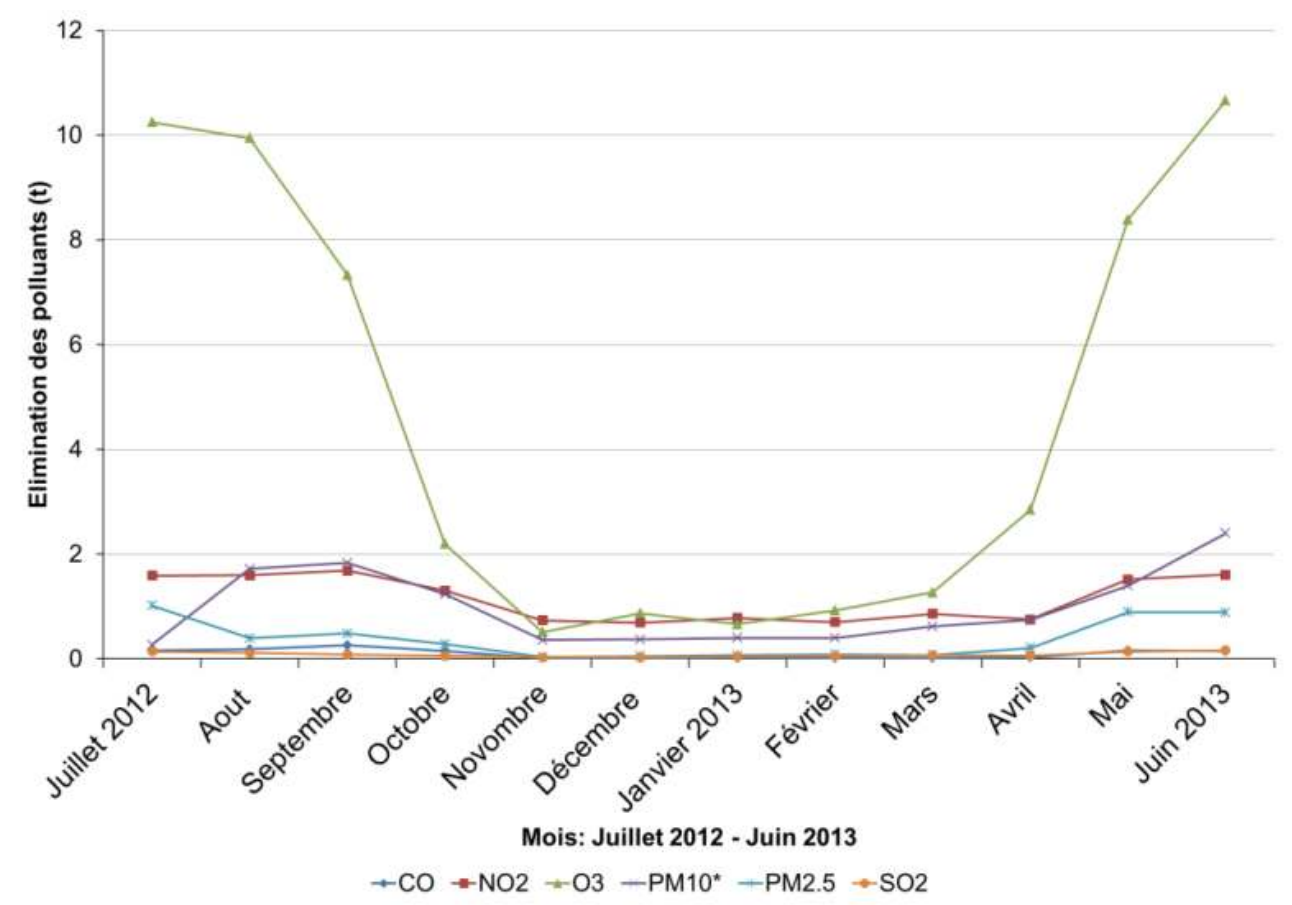

Figure 10. Élimination mensuelle des polluants atmosphériques à l'échelle de la ville de Strasbourg $\left(\mathrm{PM}_{10} * \mathrm{PM}_{10}\right.$ coarse $)($ Selmi, 2014)

Une comparaison entre le taux d'élimination des polluants par les arbres et le taux d'émissions des polluants atmosphériques à Strasbourg montre que la végétation arborée gérée par les services municipaux a éliminé environ $0.03 \%$ du CO émis, $7 \%$ des $\mathrm{PM}_{10}$ coarse, $1.5 \%$ des $\mathrm{PM}_{2.5}$ et de $0.5 \%$ du SO (Tableau $^{\circ} 4$ ). 
Tableau 4. Comparaison entre les émissions atmosphériques et l'élimination des polluants par les arbres à l'échelle de la ville de Strasbourg (Selmi, 2014)

\begin{tabular}{|l|l|l|l|}
\hline Polluants & $\begin{array}{l}\text { Taux d'élimination des } \\
\text { polluants } \\
\text { (R: }: \text { t/an) }\end{array}$ & $\begin{array}{l}\text { Émission des polluants (E : t/an) } \\
\text { (ASPA, 2014) }\end{array}$ & $\begin{array}{l}\text { Ratio (R/E } \\
\times 100)(\%)\end{array}$ \\
\hline CO & 1.2 & 3,910 & 0.03 \\
\hline NO $_{2}$ & 13.84 & $\begin{array}{l}\text { Les émissions du NO } 2 \text { ne sont pas } \\
\text { estimées. } \\
\text { NO }\end{array}=2633$ & 0.5 \\
\hline PM $_{10 \text { caarse }}$ & 11.76 & 177 & 6.6 \\
\hline PM $_{2.5}$ & 4.5 & 308 & 1.5 \\
\hline SO $_{2}$ & 1.03 & 209 & 0.5 \\
\hline
\end{tabular}

Par ailleurs, le pourcentage de l'amélioration horaire de la qualité de l'air est de l'ordre de $0.002 \%$ pour le $\mathrm{CO}, 0.43 \%$ pour le NO2, $0.57 \%$ pour l'O3, $1.05 \%$ pour les $\mathrm{PM}_{10}$ coarse, $0.2 \%$ pour les PM2.5 et $0.38 \%$ pour le SO2.

\subsection{Discussions} urbains et d'identifier de nouvelles perspectives de recherche

\subsubsection{Quelques enseignements de l'évaluation écologique des services écosystémiques}

La faculté des arbres à stocker et à séquestrer le carbone dépend de facteurs tant environnementaux (exposition à la lumière, distribution, etc.) que structuraux (âge et taille, état sanitaire, densité des arbres, surface foliaire, espèces, etc.). L'âge et la taille sont les deux facteurs structuraux déterminants dans le stockage et la séquestration du carbone. Comme l'ont suggéré les résultats, les arbres stockent et séquestrent le carbone pendant toute la phase de croissance durant laquelle les taux stockés et séquestrés ne cessent de s'accroître. Arrivés à maturité, l'aptitude des arbres à stocker le carbone diminue et ils commencent à émettre du dioxyde de carbone à long et à court terme. Dans ce cas, les gestionnaires peuvent opter pour la stratification du couvert arboré en privilégiant une présence conjointe des différentes classes d'âge et de diamètre dans les parcs et les zones semi-forestières (McPherson, 1998). Un tel recours permet aux jeunes pousses de stocker et de séquestrer rapidement le carbone émis par les arbres en décomposition.

41 Signalons que les pratiques de gestion influencent aussi le taux de carbone émis par les arbres. Pour stabiliser le flux de carbone, les gestionnaires doivent agir par exemple sur le choix des espèces rustiques, tolérantes aux conditions du milieu urbain et qui n'exigent 
pas un entretien intensif (Ginkgo, Micocoulier, Metasequoia, etc.) (Balez, 2008) ${ }^{1}$. En outre, ils peuvent éviter les espèces sensibles à la pollution et favoriser les espèces ayant une bonne qualité de bois qui pourrait être la matière première pour des équipements à base de bois (Sophora du Japon; Noisetier ; Châtaignier, Frêne, etc.) (Chapparo et Terrades, 2009). Un bois mort transformé en équipement (banc, table, chaise, etc.) garde son stock de carbone jusqu'à sa destruction tandis qu'un bois mort recyclé sous forme de papier ou brulé émet rapidement le carbone vers l'atmosphère durant tout le processus de recyclage ou de destruction (Massolia, 2011)2. Des solutions peuvent aussi concerner les outils d'entretien et de suivi en limitant le recours aux énergies fossiles.

Les arbres contribuent, même modestement, à réduire la pollution de l'air, mais ils ne sont pas la seule solution pour remédier à ce problème. Le taux d'élimination des polluants par les arbres à Strasbourg $\left(6 \mathrm{~g} / \mathrm{m}^{2}\right.$ de couvert arboré) est comparable à la moyenne d'élimination des polluants par les arbres urbains aux États-Unis $\left(6.7 \mathrm{~g} / \mathrm{m}^{2} \mathrm{de}\right.$ couvert arboré). En revanche, notre estimation est plus faible que celles trouvées à Barcelone $\left(9.35 \mathrm{~g} / \mathrm{m}^{2}\right)$, à Brooklyn $\left(10.2 \mathrm{~g} / \mathrm{m}^{2}\right)$, Los Angeles $\left(23 \mathrm{~g} / \mathrm{m}^{2}\right)$. Le potentiel des arbres à éliminer les polluants dépend de différents facteurs comme la précipitation, le couvert arboré, l'état sanitaire, la concentration de la pollution atmosphérique, la phénologie, le pourcentage du feuillage persistant dans chaque ville (Nowak, 2006).

L'élimination des polluants est aussi influencée par la taille et l'état sanitaire des arbres; Nowak (1994) a souligné qu'un arbre de grande taille et en bon état élimine 60 fois plus de polluants qu'un arbre de petite taille. Par conséquent, le maintien du patrimoine arboré existant pourrait contribuer efficacement dans l'amélioration de la qualité de l'air dans le milieu urbain. Les gestionnaires pourraient améliorer le service d'élimination des polluants en adoptant des techniques de gestion appropriées. À titre d'exemple, les pratiques de l'art topiaire (la taille des arbres pour un but esthétique) entrainent non seulement l'augmentation des émissions des composés organiques volatils par les arbres (une taille intensive soumet les arbres à un stress qui induit une augmentation des émissions des COV) et les émissions des polluants (émis par les machines de gestion), mais aussi la réduction de la surface foliaire qui est l'interface des échanges gazeux de prédilection. En outre, il est recommandé de privilégier les espèces qui tolèrent la pollution (Bouleau, Tilleul, Frêne, etc.) et d'éviter le recours à des plantations denses dans les rues étroites qui empêchent la dispersion des polluants (Wania, 2007). D'autres études ont aussi démontré la possibilité de réduire la pollution atmosphérique à l'aide de nouveaux matériaux de construction (Boonen et al, 2015 ; Angelo et al, 2013 ; etc.).

\subsubsection{Services écosystémiques et prise de décision}

En dépit de son succès, la quantification des services écosystémique a du mal à faire son entrée dans la planification urbaine. La prise en compte de leur évaluation tant écologique qu'économique pourrait être en mesure de définir des stratégies de planification en fonction des choix et des priorités politiques (Gomèz-Baggethun et al., 2013).

À titre d'exemple, notre approche d'évaluation a montré que les arbres urbains gérés par les services municipaux réduisent de $7 \%$ les émissions des particules fines. Sachant que la pollution en particules fines représente un enjeu majeur pour le développement urbain, l'estimation de l'impact des arbres sur une telle pollution pourrait aider les planificateurs à s'orienter vers l'intégration des formations végétales urbaines dans le processus de 
prise de décision en tant qu'outil pertinent pour remédier aux problèmes environnementaux urbains. Notons qu'il a été établi qu'une faible réduction des émissions de particules fines aurait un impact considérable sur le plan sanitaire et économique (Baro et al., 2014). Dans ce contexte, les nouvelles politiques de réduction des émissions atmosphériques doivent être entreprises à différentes échelles notamment, car une partie des particules est importée d'autres régions voisines.

Par ailleurs, le modèle a évalué à $38 \%$ les espaces libres qui peuvent accueillir de nouvelles plantations. Ces espaces qui sont le support de nouveaux projets d'urbanisation peuvent jouer le rôle de sites d'expérimentation à ciel ouvert pour examiner les interactions entre les pratiques de gestion, la structure de la végétation arborée mise en place et son effet physique. Une telle procédure, se focalisant à une échelle locale, n'introduit pas tous les facteurs agissant sur l'environnement urbain certes, mais elle permet de :

- modéliser la structure arborée en fonction des attentes et des services écosystémiques à valoriser

- et identifier l'impact de ces activités sur le potentiel des arbres à produire les services écosystémiques.

Cette procédure a été souvent soutenue par les chercheurs, car elle met en place une évaluation diachronique des conséquences de l'urbanisation et des nouveaux projets de planification sur le bien-être humain (Haase et al., 2014).

Un autre enseignement qui peut être tiré de l'évaluation des services écosystémiques est la disparité de distribution des services écosystémiques et d'accès aux espaces végétalisés. Cartographier ces disparités et les introduire dans le processus décisionnel pourraient remédier aux problèmes des inégalités environnementales et soutenir le développement durable urbain (Kabisch et Haase, 2014).

\subsubsection{Services écosystémiques et sciences citoyennes : vers une nouvelle ouverture}

Bien que l'évaluation des services écosystémiques urbains soit un sujet récent, de nouvelles ouvertures thématiques quant à son appréhension voient le jour. Depuis quelques années, les chercheurs s'intéressent à de nouvelles méthodes pour étudier les services écosystémiques urbains (Paavola et Hubacek, 2013). La réflexion portée sur la démocratisation des connaissances et de la prise de décision à travers la science citoyenne en est une. Cette approche se base sur la production de nouvelles formes de savoir technoscientifique et d'innovation. Considérablement utilisée dans le domaine naturaliste, la science citoyenne appliquée à l'évaluation des services écosystémiques permet l'association des efforts : i) de la recherche scientifique pour définir les objectifs de recherche et établir le protocole de collecte de données, ii) des citoyens pour l'expertise, l'observation et la collecte des données et enfin iii) de l'ensemble des parties prenantes (scientifiques, décideurs, habitants) pour définir les nouveaux projets d'aménagement (Pereira et al., 2013). Le but étant d'impliquer les citoyens dans la gouvernance locale et de les sensibiliser aux enjeux environnementaux locaux. Cette démarche induit aussi à : i) renforcer le lien entre la population et son milieu de vie, ii) éduquer et sensibiliser les citadins aux composantes naturelles urbaines et iii) prendre en compte les préférences collectives et individuelles dans l'aménagement urbain (Lovell et Taylor, 2013). 

À Londres, un projet de quantification des services écosystémiques rendus par les arbres à travers la collecte des données participative a vu le jour en 2014. Environ 200 volontaires ont été formés pour collecter les données dendrométriques dans 476 placettes. Ces données ont, par la suite, alimenté des plateformes numériques pour quantifier l'impact des arbres sur l'environnement urbain. Le deuxième atout de ce projet a été de développer une plateforme numérique qui recense et cartographie les arbres urbains en se basant sur les données collectées par la population formant ainsi une base pour de nouvelles investigations scientifiques.

\subsubsection{Limites}

51

mme toutes les approches de modélisation, le recours au modèle i-Tree Eco présente un certain nombre de limites. La première concerne l'adaptation du modèle au contexte franÇ ais. Étant développé aux États unis, une grande partie de l'architecture du modèle est fondée sur des algorithmes adaptés au contexte américain (ex. le volet consacré à la quantification de la biomasse foliaire à travers les équations allométriques). Or cette limite peut être contournée, car l'ensemble des algorithmes est ajusté en fonction des données climatologiques et atmosphériques locales et des données dendrométriques collectées sur le terrain.

La diversité des données d'entrée exigées par le modèle représente à la fois une richesse et une source d'incertitudes. Prenons l'exemple des bases de données cartographiques à partir desquelles nous avons généré notre échantillon statistique. Ces dernières font référence à des dates et des méthodes de réalisation différentes ce qui génère des incertitudes au niveau du croisement des bases de données. Par ailleurs, la base de données des espaces verts, fournie par les services techniques de la ville et qui date de 2010, n'inclut qu'une petite partie des espaces d'accompagnement et d'alignement ce qui peut engendrer une sous-estimation du couvert arboré et par conséquent des services d'élimination de polluants par exemple.

53 Enfin, le modèle a été appliqué uniquement à l'échelle des espaces végétalisés gérés par les services municipaux. Il serait donc intéressant d'étudier la strate arborée dans son ensemble (y compris les jardins privatifs) pour estimer l'effet global du patrimoine arboré à l'échelle de la ville de Strasbourg.

\section{Conclusion}

54 Le modèle a fourni une évaluation écologique quantitative et concrète de l'effet des arbres sur l'environnement urbain et de leur potentiel dans la production des services au profit de la société. Le recours à ce modèle n'a pas pour but ni de rompre les ponts avec l'évaluation économique ni de présenter ce modèle comme étant « Le » modèle unique. Nous avons plutôt cherché, d'une part, à ouvrir une nouvelle perspective dans l'appréhension du concept de "services écosystémiques "; et d'autre part, à proposer une approche préliminaire qui conduit à quantifier l'effet de la végétation arborée sur l'environnement urbain, et ce, en l'absence d'une telle démarche à l'échelle des villes françaises.

55 L'étude de l'architecture du modèle a montré qu'il regroupe et articule des modèles numériques de nature différente. De ce fait, il initie à une approche interdisciplinaire

Environnement Urbain / Urban Environment, Volume 11 | 2017 
visant à appréhender la complexité des interactions entre végétation arborée et environnement urbain.

Notre exercice d'évaluation a aussi montré que l'appréhension de la valeur instrumentale peut s'établir en ayant recours aux indicateurs biophysiques. Ces derniers ont permis de quantifier et d'analyser les $\mathrm{SE}$ en fonction des changements de l'occupation du sol. Ils ont mis en relief le potentiel de la végétation urbaine (en tant qu'écosystème) à fournir les $\mathrm{SE}$ à travers l'étude des fonctions écologiques sous-jacentes (photosynthèse, dépôt sec, métabolisme chimique, etc.).

Le recours à l'approche par habitat et l'articulation entre les indicateurs d'état et les indicateurs de performance confirme la nécessité de caractériser la végétation pour déterminer son efficacité dans la production des services écosystémiques. Elle a permis d'étudier le fonctionnement de la végétation avant de quantifier les services écosystémiques. Notre approche va dans le même sens que les conclusions émises par le rapport de Defra (2007) qui souligne que l'approche par habitat permet de mettre en place un état des lieux initial. Dans ce cadre, d'autres pistes demeurent intéressantes en proposant une étude diachronique qui s'inscrit dans une démarche à moyen et/ou à longterme. Cet exemple montre que la complexité du concept de services écosystémiques n'est pas un frein quant à son application. Comme l'indique Maris $(2013: 37):$ « Cette complexité n'est pas un obstacle en tant que tel, mais nécessite une vigilance et une prise en charge appropriée ».

Trouver une cohérence entre enjeux écologiques, sociaux et urbanistiques en milieu urbain est un engagement très présent dans le discours des acteurs, mais difficile à mettre en place en raison de la complexité du fonctionnement de l'écosystème urbain.

La détermination des politiques publiques envers l'amélioration de la qualité de vie urbaine est grandissante. La prise en compte des espaces végétalisés urbains en tant que partie intégrante multifonctionnelle de l'espace urbain devient un outil pertinent pour atteindre ces objectifs. De nombreux chercheurs soulignent l'intérêt de l'intégration des SE dans le processus de prise de décision (McDonald, 2009; Clergeau, 2012). La quantification de ces services sous leurs différents aspects (économiques, socioculturels ou écologiques) et le recours aux sciences citoyennes pourraient être, entre autres, une piste intéressante pour éclaircir l'état actuel du rapport ville-végétation.

\section{BIBLIOGRAPHY}

Abadie, J.C. (2008). La nature ordinaire face aux pressions humaines : le cas des plantes communes. Méthodes de suivis et évaluation de l'impact des activités humaines, Paris, Musée national d'histoire naturelle, $206 \mathrm{p}$.

Angelo, J., L. Andrade, L.M. Madeira et A. Mendes (2013). “An overview of photocatalysis phenomena applied to NOx abatement”, Journal of environmental management, vol. 129, p. 522-539. 
Balez, S. (2008). «Le végétal dans l'espace public urbain », École Nationale Supérieure d'Architecture de Grenoble, http://www.grenoble.archi.fr/cours-en-ligne/balez/M1CV-SB06-Le\% 20vegetal\%20dans\%20les\%20espaces\%20publics\%20urbains.pdf (consulté le 04 avril 2016)

Baro, F., L. Chaparro, E. Gomez-Baggethun, J. Langemeyer, D.J. Nowak et J. Terradas (2014). "Contribution of ecosystem services to air quality and climate change mitigation policies: The case of urban forests in Barcelona, Spain”, AMBIO, vol. 43, no 4, p. 466-479.

Bastian, O., D. Haase et K. Grunewald (2012). "Ecosystem properties, potentials and services - The EPPS conceptual framework and an urban application example”, Ecological Indicators, vol. 21, p. 7-16.

Bolund, P., et S. Hunhammar (1999). “Ecosystem services in urban areas”, Ecological Economics, vol. 29, p. 293-301.

Boonen, E., V. Akylas, F. Barmpas, A. Boréave, L. Bottalico, M. Cazaunau, H. Chen, V. Daële, T. De Marco, J.F. Doussin, C. Gaimoz, M. Gallus, C. George, N. Grand, B. Grosselin, G.L. Guerrini, H.

Herrmann, S. Ifang, J. Kleffmann, R. Kurtenbach, M. Maille, G. Manganelli, A. Mellouki, K. Miet, F. Mothes, N. Moussiopoulos, L. Poulain, R. Rabe, P. Zapf et Beeldens A (2015). "Construction of a photocatalytic De-polluting field site in the Leopold II tunnel in Brussels", Journal of Environmental Management, vol. 155, p. 136-144.

Haase, D ., N. Larondelle, E. Andersson, M. Artmann, S. Borgström, J. Breuste, E. GomezBaggethun, A. Gren, Z. Hamstead, R. Hansen, N. Kabisch, P. Kremer, J. Langemeyer, E. Lorance Rall, T. McPhearson, S. Pauleit, S. Qureshi, N. Schwarz, A. Voigt, D. Wurster et T. Elmqvist (2014). "A Quantitative Review of Urban Ecosystem Service Assessments: Concepts, Models, and Implementation", AMBIO, vol. 43, p. 413-433.

Chaparro, L., et J. Terradas J. (2009). Ecological Services of Urban Forest in Barcelona, Barcelone, Centre de Recerca Ecològica, 96p.

Clergeau, P. (2007). Une écologie du paysage urbain, Rennes, Ed. Apogée, 136 p.

Clergeau, P. (2012). « Services écologiques et Trame Verte Urbaine », VertigO - la revue électronique en sciences de l'environnement, Hors-série 12, http://vertigo.revues.org/11834 (consulté le 6 avril 2016).

de Groot, R., M.A. Wilson et R.M.J. Boumans (2002). “A typology for the classification, description and valuation of ecosystem functions, goods and services", Ecological Economics, vol. 41, no 3, p. 393-408.

de Groot, R., J. Van der Perka, A. Chiesura et A. Van Vliet (2003). "Importance and threat as determining factors for criticality of natural capital", Ecological Economics, vol. 44, no 2-3, p. 187-204.

de Groot, R., R. Alkemade, L. Braatc, L. Heina et L. Willemena (2010). “Challenges in integrating the concept of ecosystem services and values in landscape planning, management and decision making", Ecological Complexity, vol. 7, no 3, p. 260-272.

DEFRA. (2007). "Case study to develop tools and methodologies to deliver an ecosystem approach, Department for Environment, Food and Rural Affairs, http://randd.defra.gov.uk/Default.aspx? Menu=Menu\&Module=More\&Location=None\&Completed=0\&ProjectID=14753 (consulté le 07 avril 2016)

Dobbs, C., F.J. Escobedo, C. Wayne et W.C. Zipperer (2011). “A framework for developing urban forest ecosystem services and goods indicators”, Landscape and Urban Planning, vol. 99, no 3-4, p. 196-206. 
Gómez-Baggethun, E., A. Gren A, D.N. Barton, J. Langemeyer, T. McPhearson, P. O’Farrell, E. Andersson, Z. Hamstead et P. Kremer (2013). "Urban Ecosystem Services” in ELMQVIST, T., M. Fragkias, J. Goodness, B. Güneralp, P.J. Marcotullio, R.I. McDonald, S. Parnell, M. Schewenius, M. Sendstad, K.C. Seto et C. Wilkinson (Ed.), Urbanization, Biodiversity and Ecosystem Services : Challenges and Opportunities. New York-London, Springer, p. 175-251.

Haines-Young, R., et M. Potschin (2006). "Defining and identifying environmental limits for sustainable development”, University of Nottingham, http://www.nottingham.ac.uk/cem/pdf/ NR0102_FTR_Final.pdf (consulté le 7 avril 2016)

Haines-Young, R., et M. Potschin (2010). “The links between biodiversity, ecosystem services and human well-being” in Raffaelli D., et C. Frid (Ed.), Ecosystem Ecology : a new synthesis, Cambridge, Cambridge University Press, p. 110-139.

Hawkins, K. (2003). “Economic valuation of ecosystem services”, University of Minnesota http:// $\mathrm{mn} . \mathrm{gov} / \mathrm{frc} /$ documents/council/landscape/SE\%20Landscape/

MFRC_Economic_Valuation_EcosystemServices_SE_2003-10-01_Report.pdf (consulté le 1er mai 2017)

Jackson, R.B , J.T. Randerson, J.G. Canadell, R.G. Anderson, R. Avissar, D.D. Baldocchi, G.B. Bonan, K. Caldeira, N.S. Diffenbaugh, C.B. Field, B.A. Hungtae., E.G. Jobbagy, L.M. Kueppers, M.D. Nosetto et D.E. Pataki (2008). « Protecting climate with forests », Environmental research letters, vol. 3, p. 1-5.

James, P., K. Tzoulas, M.D. Adams, A. Barber, J. Box, J. Breuste, T. Elmqvist, M. Frith, C. Gordon, K.L. Greening, J. Handley, S. Haworth, A.E. Kazmierczak, M. Johnston, K. Korpelam, M. Moretti, J. Niemelä, S. Pauleit, M.H. Roe, J.P. Sadler et C. Ward Thompsons (2009). “Towards an integrated understanding of green space in the European built environment”, Urban Forestry \& Urban Greening, vol. 8, no 2, p. 65-75.

Jim, C.Y. (2011). "Holistic research agenda for sustainable management and conservation of urban woodlands", Landscape and Urban Planning, Landscape and Urban Planning, vol. 100, no 4, p. 375-379.

Kabisch, N., et D. Hasse (2014). “ Green justice or just green ? Provision of urban green spaces in Berlin, Germany”, Landscape and Urban Planning, vol. 122, p. 129-139.

Larrère, C. (1997). Les philosophies de l'environnement, Paris, Presses universitaires de France, $124 \mathrm{p}$.

Lovell, S., et J. Taylor (2013). "Supplying urban ecosystem services through multifunctional green infrastructure in the United States”, Landscape Ecol, vol. 28, no 8, p. 1447-1463.

Maris, V. (2016), Nature à vendre. Les limites des services écosystémiques, Versailles, Éditions Quae, $94 \mathrm{p}$.

McDonald, R. (2009). "Ecosystem service demand and supply along the urban-to-rural gradient", Journal of Conservation Planning, vol. 5, p. 1-14.

McPherson, E.G. (1998). “Atmospheric carbon dioxide reduction by Sacramento's urban forest”, Journal of Arboriculture, vol. 24, no 4, p. 215-223.

Massolia, (2011). « Les arbres peuvent-ils compenser les émissions de CO2 des voitures? », Massolia, http://www.massolia.com/environnement1/les-arbres-peuvent-ils-compenser-lesemissions-de-co2-des-voitures/ (consulté le 4 avril 2016)

Nowak, D. (1994). “Atmospheric Carbon Dioxide Reduction by Chicago's Urban Forest”, in :

McPherson E.G, Nowak D.J., Rowntree R.A., et Rowan A. (eds.), Chicago's Urban Forest Ecosystem : 
Results of the Chicago Urban Forest Climate Project, Radnor, Northeastern Forest Experiment Station, p. 83-94.

Nowak, D.J., et D. Crane (2000). “The Urban Forest Effects (UFORE) model : quantifying urban forest structure and functions, in Hansen M., Brk T. (dir), Integrated Tools for Natural Resources Inventories in the 21st Century, coll. Integrated Tools proceedings, p. 714-720.

Nowak, D.J., D.E. Crane et J.C Stevens (2006). “Air pollution removal by urban trees and shrubs in the United States”, Urban Forestry and Urban Greening, vol. 4, no 2-3, p. 115 -123.

Nowak, D.J., J.T. Walton, J.C. Stevens, D.E. Crane et R.E. Hoehn (2008). "Effect of plot and sample size on timing and precision of urban forest assessments", Arboriculture and Urban Forestry, vol 34, p. 386-390.

Paavola, J., et K. Hubacek (2013). “Ecosystem Services, Governance, and Stakeholder Participation: An Introduction", Ecology and Society, vol. 18, no 4, p. 1-6.

Paoletti, E., T. Bardelli, G. Giovannini et L. Pecchioli (2011). "Air quality impact of an urban park over time", Procedia Environmental Sciences, vol. 4, p. 10-16.

Pereira, H.M., S. Ferrier, M. Walters, G.N. Geller, R.H.G. Jongman, R.J. Scholes, M.W. Bruford, N. Brummitt, S.H.M. Butchart, A.C. Cardoso, N. C. Coops, E. Dulloo, D. P. Faith, J. Freyhof, R. D. Gregory, C. Heip, R. Höft, G. Hurtt, W. Jetz, D.S. Karp, M. A. McGeoch, D. Obura, Y. Onoda, N. Pettorelli, B. Reyers, R. Sayre, J. P. W. Scharlemann, S. N. Stuart, E. Turak, M. Walpole et M. Wegmann (2013). “Essential biodiversity variables”, Science, vol. 339, p. 277-278.

Pickett, S.T.A., M.L. Cadenasso, J.M. Grove, C.H Nilon, R.V. Pouyat, W.C. Zipperer et R. Costanza R (2008). "Urban Ecological Systems : Linking Terrestrial Ecological, Physical, and Socioeconomic Components of Metropolitan Areas", in Marzluff J.M., E. Shulenberger, W. Endlicher, M. Alberti, G. Bradley, C. Ryan, U. Simon et C. ZumBrunnen (Ed.), Urban Ecology, New-York, Springer US, p. 99-122.

Ryan, M.G., M.E. Harmon, R.A. Birdsey, P. Giardina, L.S. Heath, R.A. Houghton, R.B. Jackson, D.C. Mckinley, J.F. Morrison, B.C. Murray, D.E. Pataki et K.E. Skog (2010). “A synthesis of the science on forest and carbon for U.S. forests", Issues Ecology, vol. 13, p. 1-16.

Rogers, K., T. Jarrat et D. Hansford (2011). « Torbay's Urban forest : Assessing urban forest effects and values », Torbay, Treeconomics, http://www.itreetools.org/resources/reports/ Torbay_UF_Report.pdf (consulté le 4 avril 2016)

Sauveur, B., (2010). « De la loi naturelle à la deep ecology : les ambiguités de la nature », Revue d'éthique et de théologie morale, no 261, p. 235-242.

Selmi, W. (2014). Services écosystémiques rendus par la végétation urbaine. Application d'approches d'évaluation à la ville de Strasbourg, Strasbourg, Université de Strasbourg, 330 p.

Teyssèdre, A. (2010). « Les services écosystémiques, notion clé pour explorer et préserver le fonctionnement des (socio)écosystèmes ", Société FranÇ aise d'Écologie, http:// www.sfecologie.org/regards/2010/10/25/regards-4-teyssedre/ (consulté le04 avril 2016)

Tietenberg, T.H., L. Lewis et P. Naccache (2013). Économie de l'environnement et développement durable, New Jersey, Pearson, $412 \mathrm{p}$.

UNFPA. (2007). État de la population mondiale 2007 libérer le potentiel de la croissance urbaine ? New-York. Fonds des Nations Unies pour la population, $99 \mathrm{p}$.

Vallet, J. (2009). Gradient d'urbanisation et communautés végétales d'espaces boisés Approche à plusieurs échelles dans trois agglomérations du Massif armoricain, Angers, Université d'Angers, $292 \mathrm{p}$. 
Young, R.F. (2010). “Managing municipal green space for ecosystem services”, Urban Forestry and urban greening, vol. 9, no 4, p. 313-321.

\section{ABSTRACTS}

This paper addresses both the conceptual complexity and the pragmatic aspect of the ecosystem services concept. By providing a concrete example of assessing ecosystem services, the purpose of this paper is to demonstrate that this complexity is not an obstacle to the application of the concept. In this context buttom-up approach has been developed. Through the characterization of urban vegetation, this ecological assessment studied the functioning of urban trees and quantified ecosystem services using "i-Tree Eco model " in Strasbourg city, France.

Cet article aborde à la fois la complexité du concept de services écosystémiques et son aspect pragmatique. En fournissant un exemple concret d'évaluation de services écosystémiques, l'objectif est de démontrer que cette complexité n'est pas un frein quant à l'application du concept. Dans ce cadre, une évaluation ascendante des services écosystémiques a été développée. Basée sur la caractérisation de la végétation urbaine, cette évaluation écologique a permis d'étudier le fonctionnement des arbres urbains et de quantifier les services écosystémiques rendus à la société en appliquant le modèle «i-Tree Eco » à l'échelle de la ville de Strasbourg (France).

\section{INDEX}

Keywords: urban trees, ecosystem services, functions, assessment, i-Tree Eco

Mots-clés: Arbres urbains, services écosystémiques, fonctions, évaluation, i-Tree Eco

\section{AUTHORS}

\section{WISSAL SELMI}

Docteure en Géographie, docteur chargée de projet « services écosystémiques », ApiTrees, 2 rue Henri Lemaire, Frasnes-Lez-Anvaing, Belgique.), courriel : wissalselmi@yahoo.fr

\section{CHRISTIANE WEBER}

Géographe, directrice de recherche, UMR TETIS (CNRS, AgroParis Tech, Cirad, Irstea) Uni. Montpellier, Montpellier, France, courriel : christiane.weber@teledetection.fr 